\title{
Entanglement-free Heisenberg-limited phase estimation
}

\author{
B. L. Higgins, ${ }^{1}$ D. W. Berry, ${ }^{2}$ S. D. Bartlett,${ }^{3}$ H. M. Wiseman,${ }^{1,4}$ and G. J. Pryde ${ }^{1}$ \\ ${ }^{1}$ Centre for Quantum Dynamics, Griffith University, Brisbane 4111, Australia \\ ${ }^{2}$ Centre for Quantum Computer Technology, Macquarie University, Sydney 2109, Australia \\ ${ }^{3}$ School of Physics, University of Sydney, Sydney 2006, Australia \\ ${ }^{4}$ Centre for Quantum Computer Technology, Griffith University, Brisbane 4111, Australia
}

Measurement underpins all quantitative science. A key example is the measurement of optical phase, used in length metrology and many other applications. Advances in precision measurement have consistently led to important scientific discoveries. At the fundamental level, measurement precision is limited by the number $N$ of quantum resources (such as photons) that are used. Standard measurement schemes, using each resource independently, lead to a phase uncertainty that scales as $1 / \sqrt{N}$ - known as the standard quantum limit. However, it has long been conjectured [1, 2] that it should be possible to achieve a precision limited only by the Heisenberg uncertainty principle, dramatically improving the scaling to $1 / N$ [3]. It is commonly thought that achieving this improvement requires the use of exotic quantum entangled states, such as the NOON state [4, 5]. These states are extremely difficult to generate. Measurement schemes with counted photons or ions have been performed with $N \leq 6[6,7,8,9,10,11,12,13,14,15$, but few have surpassed the standard quantum limit [12, 14] and none have shown Heisenberg-limited scaling. Here we demonstrate experimentally a Heisenberg-limited phase estimation procedure. We replace entangled input states with multiple applications of the phase shift on unentangled single-photon states. We generalize Kitaev's phase estimation algorithm [16] using adaptive measurement theory [17, 18, 19, 20] to achieve a standard deviation scaling at the Heisenberg limit. For the largest number of resources used $(N=378)$, we estimate an unknown phase with a variance more than $10 \mathrm{~dB}$ below the standard quantum limit; achieving this variance would require more than 4,000 resources using standard interferometry. Our results represent a drastic reduction in the complexity of achieving quantumenhanced measurement precision.

Phase estimation is a ubiquitous measurement primitive, used for precision measurement of length, displacement, speed, optical properties, and much more. Recent work in quantum interferometry has focused on $n$ photon NOON states [5, 6, 7, 8, 9, 10, 11, 12, 21, $(|n\rangle|0\rangle+|0\rangle|n\rangle) / \sqrt{2}$, expressed in terms of number states of the two arms of the interferometer. With this state, an improved phase sensitivity results from a decrease in the phase period from $2 \pi$ to $2 \pi / n$. We achieve improved phase sensitivity more simply using an insight from quantum computing. We apply Kitaev's phase estimation algorithm [16, 22] to quantum interferometry, wherein the entangled input state is replaced by multiple passes through the phase shift. The idea of using multi-pass protocols to gain a quantum advantage was proposed for the problem of aligning spatial reference frames [23, and further developed in relation to clock synchronization 24] and phase estimation [25, 26].

The conceptual circuit for Kitaev's phase estimation algorithm is shown in Fig. 19. The algorithm yields, with $K+1$ bits of precision, an estimate $\phi_{\text {est }}$ of a classical phase parameter $\phi$, where $e^{i \phi}$ is an eigenvalue of a unitary operator $U$. It requires us to apply $K+1$ unitaries, $U^{p}$, with $p=2^{K}, 2^{K-1}, \ldots, 1$, each controlled by a different qubit. Each qubit is prepared in the state $H|0\rangle=$ $\frac{1}{\sqrt{2}}(|0\rangle+|1\rangle)$, and the control induces a phase shift $e^{i p \phi}$ on the $|1\rangle$ component. The qubits are measured sequentially in the $\hat{\boldsymbol{\sigma}}_{x}$ basis $(X)$, and the results control additional phase shifts, indicated by $R(\alpha) \equiv \exp (i \alpha|0\rangle\langle 0|)$, on subsequent qubits. This enables the inverse quantum Fourier transform to be performed without entangling gates [27. With a random phase $\theta$ on the qubits, as shown in Fig. 17, the measurement results on the qubits are the binary digits of $\left(\phi_{\text {est }}-\theta\right) / 2 \pi$; this ensures that the accuracy of the estimate is independent of the value of $\phi$.

Alternatively, this independence could be obtained by using a second classical "feedback" phase $\theta$, as in Fig. 1 b, which also eliminates the need for many of the gates in Fig. 1 1 a. This is a classical real-valued parameter whose value is adjusted by $\pi / p$, indicated by the symbol $D(\pi / p)$, controlled by the results of measurements. The value of $\theta$ determines (as indicated by the diamondshaped control symbol in Fig. 1 $\mathrm{b}$ ) phase-shifts $R(p \theta)$ on the qubits. Applying this to interferometry, we can measure an unknown optical phase $\phi$ using dual-rail photonic qubits $[22$. Here the operator $U$ induces a relative phase shift $\phi$ each time the beam path (in one arm of the interferometer) passes through the unknown optical phase $\phi$. The additional phase shifts (determined by $\theta$ ) can be implemented using a single-pass controllable phase in the other arm.

If a fixed probability of error in $\phi_{\text {est }}$ is allowed (that is, if the uncertainty is quantified by a confidence interval), then the uncertainty of Kitaev's phase estimation scales as $2^{-K}[22$. Because the number of control photons is $N_{\text {phot }}=K+1$, this scaling implies an exponential decrease in the phase uncertainty with increas- 
a

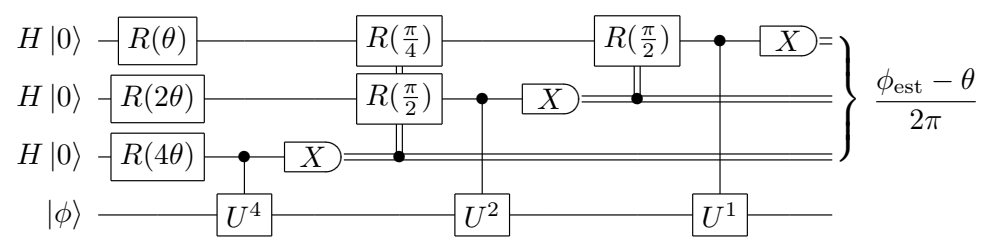

b

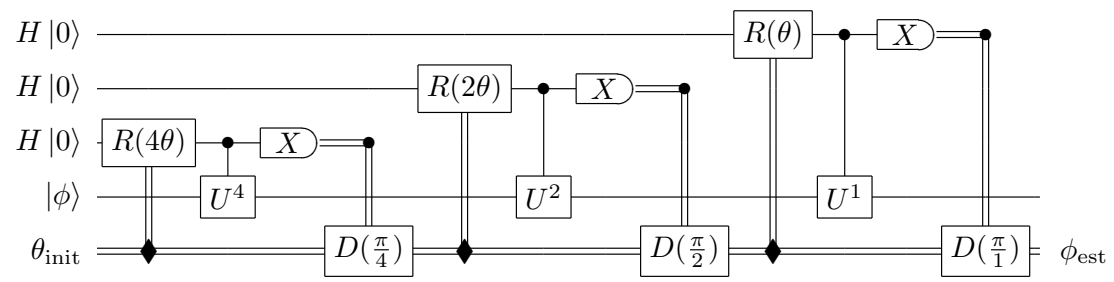

c

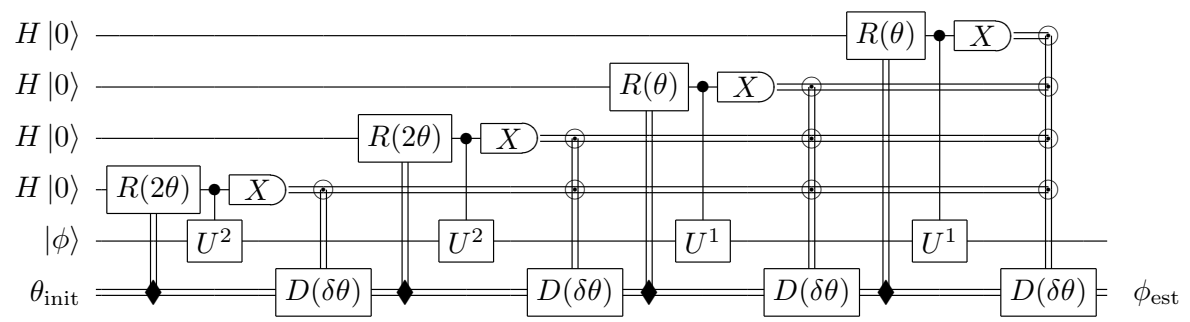

FIG. 1: Quantum circuit diagrams of Kitaev's phase estimation algorithm and our generalization. a, Kitaev's algorithm 16. with the inverse quantum Fourier transform implemented with measurement and classical feedback 27. and a random initial phase estimate $\theta$. In general, $K+1$ qubits yield $K+1$ binary digits of precision; here $K=2$. b, As in a, but here we implement $\theta$ (now called $\theta_{\text {init }}$ ) and the feedback operations by coupling the qubits to a common element, the "feedback phase" $\theta$ (the lowest rail). c, Generalization of the circuit to include $M \geq 1$ qubits for each binary digit; here $K=1$ and $M=2$. For details on circuit elements, see text.

ing resources - apparently violating the Heisenberg uncertainty principle. The correct analysis, however, is as follows. Although the cost of implementing $U^{p}$ can be assumed to be essentially independent of $p$ in the context of quantum computation, in interferometry it requires $p$ applications of the phase shift, and should thus be counted as requiring $p$ resources 25. Using this definition, the total number of resources used is $N=2^{K+1}-1$. Then for $N \gg 1$, the uncertainty scales as $1 / N$, as in the Heisenberg limit. We note that this quantification of resources in terms of the number of applications of the phase shift is the relevant one for phase estimation of sensitive (for example, biological) samples, wherein the goal is to pass as little light through the sample as is necessary.

On the other hand, if $\Delta \phi_{\text {est }}$ is taken to be the standard deviation - the usual measure of uncertainty - then Kitaev's algorithm does not scale as $1 / N$. Rather, we have shown analytically that it asymptotes as $\sqrt{2} / \sqrt{N}$, the same scaling as the standard quantum limit (SQL) see also Ref. [21]. The broad wings of the distribution of phase estimates are not due to any deficiency in the estimation procedure - the quantum Fourier transform is optimal-but rather are a consequence of the sequence of phase shifts on the photons, $2^{K} \phi, 2^{K-1} \phi, \ldots, \phi$.

A key idea to address this problem is to employ
$M$ copies of the control photon at each phase shift 23, 24, 25]. For $M>1$ one cannot perform an exact quantum Fourier transform using only single-photon operations. However, one can perform it approximately using the adaptive phase estimation scheme of Ref. [18], as shown in Fig. 1 for $M=2$. Here the feedback phase is adjusted by an amount $\delta \theta$, controlled by all previous measurement results via a bayesian algorithm. This general multi-bit conditioning is represented by the circled-dot symbol in Fig 1 . For the final adjustment, $\delta \theta=\phi_{\text {est }}-\theta$, so the value of $\theta$ that is read out is equal to $\phi_{\text {est }}$, the best estimate of the phase given all the data, as in Fig. 1 b. Because the inverse quantum Fourier transform is not performed exactly, and because the sequence of phase shifts is not exactly equivalent to the optimal state of Ref. [18], we do not expect $\phi_{\text {est }}$ to have an uncertainty precisely at the Heisenberg limit $\Delta \phi_{\mathrm{HL}}=\tan [\pi /(N+2)] \approx \pi / N$ for $N \gg 1$ [18, 28]. Nevertheless, our algorithm allows estimation with an uncertainty only a constant factor larger than this ultimate limit, for $M \geq 4$. For instance, we find by numerical simulation that for $M=6$, $\Delta \phi_{\mathrm{est}} \approx 1.56 \pi / N$ for $N \gg 1$.

A conceptual implementation of this generalization of Kitaev's algorithm is shown in Fig. 2. It works as follows: a photon is converted to the state $\frac{1}{\sqrt{2}}(|1\rangle|0\rangle+|0\rangle|1\rangle)$ 


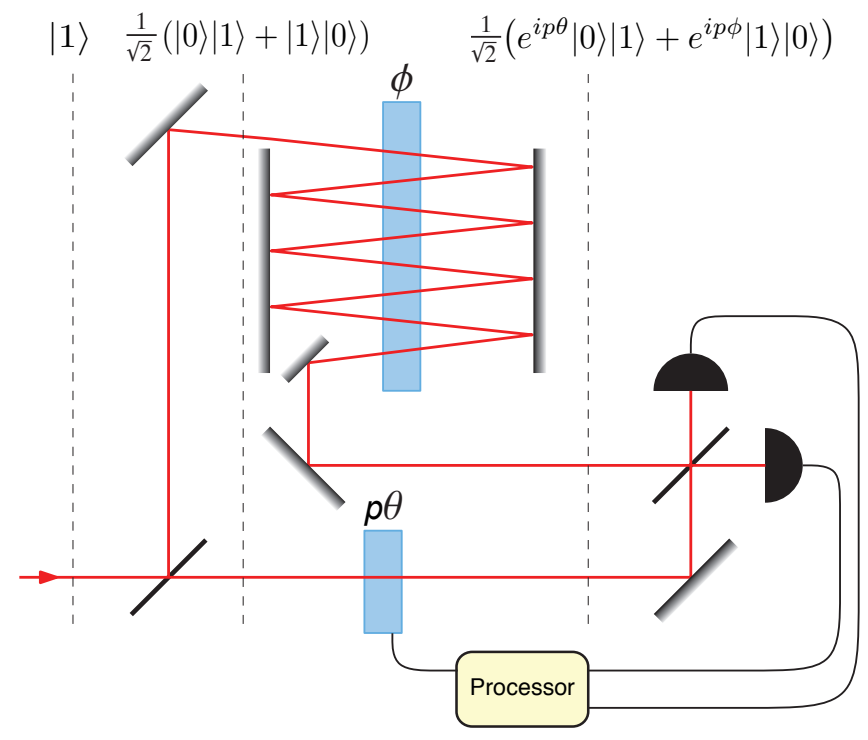

FIG. 2: Conceptual diagram of the algorithm's implementation as a Mach-Zehnder interferometer. This is equivalent to the scheme in Fig. 1k. Photon-number quantum states are shown at key points. The first beam splitter implements the Hadamard operation on incident photons. The large phaseshift element is configured to implement a $p \phi$ phase shift on logical $|1\rangle$ states, with $p$ adjustable ( $p=8$ shown). The small phase-shift element implements the adjustable $p \theta$ phase shift on logical $|0\rangle$ states. The final beam splitter and single photon detectors implement a $\hat{\boldsymbol{\sigma}}_{x}$ measurement, which determines, via the processor, how to adjust $\theta$ before the next photon input.

by the first beam splitter. After passing $p=2^{K}$ times through the phase shift $\phi$, the state evolves to $\frac{1}{\sqrt{2}}\left(e^{i p \theta}|1\rangle|0\rangle+e^{i p \phi}|0\rangle|1\rangle\right)$. The photon is then detected after the modes are recombined on the second beam splitter. The result is used to update the probability distribution $P(\phi)$ which represents knowledge about $\phi$.

This process is repeated $M$ times, so that $M$ independent photons go through $2^{K}$ passes in sequence. Quantifying a resource as a single pass of a photon through the phase shift, each photon in this stage corresponds to $2^{K}$ resources. Following these $M$ photons, another $M$ photons undergo the same process at $p=2^{K-1}$ passes, and so on for $p=2^{K-2}, \ldots, 2^{0}$. Thus a total of $M(K+1)$ photons and $N=M\left(2^{K+1}-1\right)$ resources are used. The value of the feedback phase $\theta$ is random for the first photon only. Thereafter it is chosen, based upon $P$ (that is, upon all preceding results), to minimize the expected phase variance after the next detection [18.

This bayesian control algorithm reduces to Kitaev's algorithm for $M=1$. We have shown analytically that this algorithm gives a standard deviation of estimates scaling as the SQL for $M=2$ as well as $M=1$, but numerical simulations (for $N$ up to $4 \times 10^{6}$ and $M$ up to 8) demonstrate a Heisenberg-limited scaling for $M \geq 4$.

We note that a single photon with $p$ passes through the unknown phase shift is operationally equivalent to a
NOON state with $n=p$ photons, and involves exactly the same number of resources. A single NOON state such as this yields at most one bit of information [4, and only about $\phi$ modulo $2 \pi / n$. It has been shown numerically 21] that a sequence of NOON states, with $n$ as well as $\theta$ chosen adaptively, achieves Heisenberg-limited scaling, but only for $N>100$. Our generalized algorithm, which is simpler, can also be applied to NOON states, and directly achieves Heisenberg-limited scaling. Even if high- $n$ NOON states could be produced, however, they require high- $n$ photon-number-resolving detectors, and are proportionately more sensitive to detector inefficiency than single photons with multiple passes through the phase shift.

We demonstrate our single-photon algorithm using a common-spatial-mode polarization interferometer, as shown in Fig. 3 Common-spatial-mode interferometers are used in many metrology tasks involving birefringent materials such as stress sensors, Faraday spectroscopy, and testing optical components, but we stress that our algorithm applies equally well to any interferometer. The two arms of the interferometer are the right-circular $(|R\rangle)$ and left-circular $(|L\rangle)$ polarization modes. The unknown phase $\phi$ is implemented as a birefringent half-wave plate. We test two versions of the algorithm: $M=1$ (Kitaev's algorithm), and $M=6$ (chosen for its robustness). For each, we vary the number of resources $N=M\left(2^{K+1}-1\right)$ by choosing different values for the maximum number of passes, $2^{K}$, with $K \in\{0,1,2,3,4,5\}$. We also measured the standard deviation for a non-adaptive or "standard" estimation algorithm, using $N$ single passes of the phase shift, with $N$ chosen equal to the number of resources used for each of the $M=6$ data points. In this case $\theta$ was incremented non-adaptively [29] by $\pi / N$ from one photon to the next, to ensure a sensitivity independent of $\phi-\theta_{\text {init }}$.

The experimental results are shown in Fig. 4 together with theoretical calculations. The error bars are $95 \%$ confidence intervals determined using a studentized bootstrap on a $\log$ scale [30. In general, the distributions have a large positive kurtosis which emphasizes the effect of outliers; our error calculation takes this into account to provide accurate error bars. Theoretical predictions assume $100 \%$ visibility. Experimentally, visibilities for $p=1$ to 16 were high (all above $98.1 \%$ and typically above $99.6 \%$ ). However the $p=32$ case had slightly lower visibility $(95.4 \%)$, leading to higher than expected standard deviations for the $N=378$ case. This is primarily due to expansion of the beam, with consequent overlap of beams, leading to a small probability of measuring the photon after only 30 passes.

The results of the non-adaptive phase estimation algorithm follow the SQL, as expected. We note that the standard deviations of the $M=1$ (Kitaev's) case also follow an SQL scaling. Most importantly, there is a clear Heisenberg scaling, $\Delta \phi_{\text {est }} \propto 1 / N$, of our adaptive multi-pass algorithm for $M=6$. Our data are consistent with the predicted overhead factor of 1.56 relative 


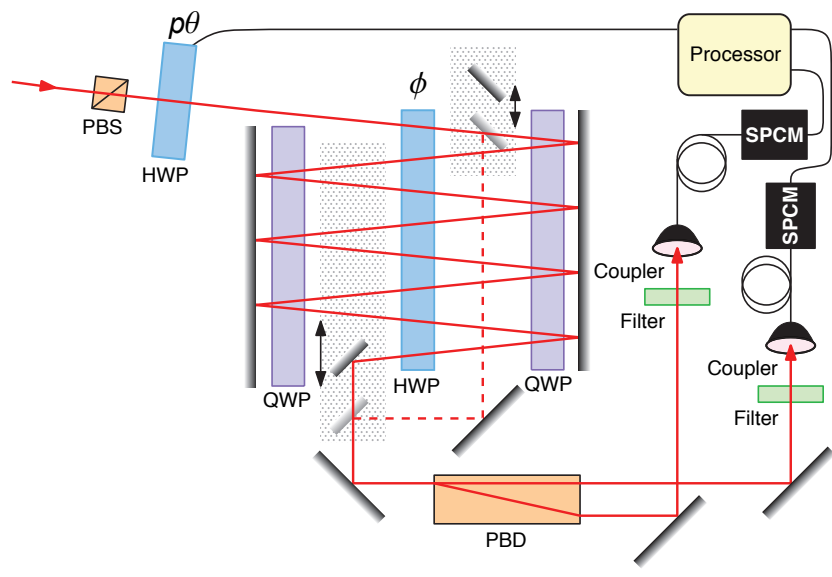

FIG. 3: Schematic of the experiment. Polarization modes replace the arms of the interferometer in Fig. 2 with phase shifts implemented by half-wave plates (HWPs). A photon experiences phase shifts between left- and right-circular polarizations by the feedback wave plate $(p \theta)$ and the unknown phase wave plate $(\phi)$. The photon is selected by a mirror mounted on a motorized translation stage, discriminated in the horizontal/vertical polarization basis by a polarizing beam displacer (PBD), and passed through a 10-nm-bandwidth interference filter. It is then coupled into a multimode fibre and detected by a single-photon counting module (SPCM), completing the $\hat{\boldsymbol{\sigma}}_{x}$ measurement. PBS, polarizing beam splitter; QWP, quarter-wave plate.

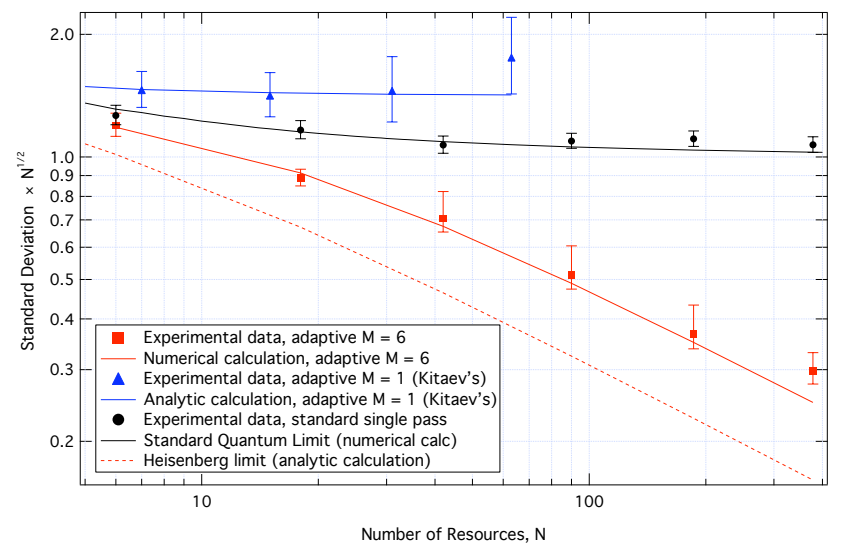

FIG. 4: Standard deviations of distributions of phase estimates for varying numbers of resources $N$. We compare theoretical predictions (lines) and measured values (points, each representing 1,000 estimates) for standard phase estimation and our implementation of Kitaev's $(M=1)$ and generalized Kitaev's $(M=6)$ algorithms. Error bars denote $95 \%$ confidence intervals. Our algorithm clearly has a lower standard deviation in phase estimates than both the SQL and Kitaev's algorithm (which has SQL scaling). For large $N$, the curve for the adaptive algorithm is parallel to the Heisenberg limit, with a small overhead factor of about 1.56. to the asymptotic Heisenberg-limited standard deviation $\pi / N$. Despite this constant overhead, our phase estimates clearly surpass the SQL. For example, where we have demonstrated the use of 378 resources in our algorithm (corresponding to a maximum of 32 passes), 4,333 resources would be required using standard techniques to achieve the same uncertainty.

We have introduced a new algorithm for phase estimation, generalizing Kitaev's algorithm, which requires no entanglement to achieve Heisenberg-limited scaling independent of $\phi$. Our algorithm uses single-photon Fock states, multiple passes and adaptive measurement. We have used our algorithm to successfully demonstrate the first measurement with Heisenberg-limited scaling. This technique has promise for a wide range of metrology tasks, especially in light of continued development of high-flux single photon sources and efficient detectors.

\section{Methods Summary}

Interferometer. A spontaneous parametric downconverter supplies pairs of single photons, one to the interferometer and one to an independent detector. The state after the polarizing beam splitter is $(|R\rangle+$ $|L\rangle) / \sqrt{2}$, equivalent to the logical state $H|0\rangle$. A photon passing through the 50 -mm-diameter $\phi$ phase shift half-wave plate undergoes a polarization rotation: $\frac{1}{\sqrt{2}}(|R\rangle+|L\rangle) \rightarrow \frac{1}{\sqrt{2}}\left(|R\rangle+e^{i \phi}|L\rangle\right)$ for a half-wave plate setting of $\phi / 4$. Two 50-mm-diameter mirrors are placed on either side, allowing a single photon to pass through the half-wave plate multiple times. To correct the unwanted $\pi$-phase shift (in the $|H\rangle /|V\rangle$ basis) on reflection, a quarter-wave plate, set to its optic axis, is inserted before each of the large mirrors. The feedback phase is implemented as another half-wave plate mounted in a computer-controlled rotation stage before the unknown phase and mirrors. We use a fixed phase $\phi$, but the use of a uniformly distributed random initial feedback phase is equivalent to performing the protocol over the full range of system phases, $\phi \in[0,2 \pi)$. Mirrors on computercontrolled translation stages are used to select the $2^{k}$ th pass for each value of $k$. Measurement is performed in the horizontal/vertical basis, corresponding to a $\hat{\boldsymbol{\sigma}}_{x}$ measurement, with a high-contrast-ratio calcite polarizing beam displacer. The two outputs of the beam displacer, filtered with 10-nm-bandwidth filters to reject background light, are sent to single photon counting modules. A successful measurement is heralded by a coincidence between the directly detected photon and either output detector.

Statistics for phase. For a phase $\phi$ with distribution $P$, an appropriate measure of error in the estimate $\phi_{\text {est }}$ is $\left\langle\cos \left(\phi-\phi_{\text {est }}\right)\right\rangle_{P}^{-2}-1$. This achieves its minimum, the Holevo variance $V_{\mathrm{H}}\left[28\right.$, for $\phi_{\text {est }}=\arg \left(\langle\exp (i \phi)\rangle_{P}\right)$, which is the estimate we use. When applied to a phase the terms variance and standard deviation are to be understood as $V_{\mathrm{H}}$ and $\sqrt{V_{\mathrm{H}}}$ respectively. 


\section{Methods}

Source. Our type-I BiBO (bismuth borate) spontaneous parametric down-conversion source is pumped by a frequency-doubled mode-locked Ti:sapphire laser, producing pairs of 820-nm, 2-nm-bandwidth single photons in the state $|H H\rangle$. One photon is guided to the experiment through a single-mode optical fibre, the other is guided straight to a single-photon counting module. A successful measurement is heralded by a coincidence between the directly detected photon and either of the output detectors - coincidence detection reduces background and dark counts, ensuring high-fidelity conditional single photons in the experiment.

Quarter-wave plate setting. For logistical reasons, we use 25-mm-diameter quarter-wave plates for most experiments, but 50 -mm-diameter wave plates for the $K=5$ cases. The wave plates are nominally identical except for their diameter. The quarter-wave plates did introduce a technical challenge: because of the largediameter optics, and the need to use all of the clear aperture to obtain multiple reflections, we used mountings that did not allow easy calibration and adjustment of the quarter-wave plates. This in turn led to small additional phase shifts from the quarter-wave plates that were dependent upon the number of passes. This problem is easily modelled and is not fundamental.

Analytic solutions. For the cases $M=1$ (Kitaev) and $M=2$ of our algorithm we have shown analytically that the variance scales as the SQL, by solving the adaptive scheme exactly, using the formulae in Ref. [18. The exact results for the variances are $2 / N+1 / N^{2}$ and $2 / N$ respectively.

Error calculation. The $95 \%$ confidence intervals shown were determined using a studentized bootstrap on a log scale. The bootstrap is a method of determining confidence intervals without making assumptions about the form of the underlying distribution [30. The data are used as a model of the underlying distribution, and confidence intervals for the quantity of interest are estimated by sampling from this distribution. That is, a number of subsamples equal to the size of the data set, $m$, is obtained, and an estimate of the quantity of interest is determined from this set of subsamples. A large number of bootstrap samples are used (in our case $10^{6}-1$ ), where each sample is an estimate of the quantity of interest based on the set of $m$ subsamples. The distribution obtained for the quantity of interest is then used to determine a confidence interval, as described on page 199 of Ref. 30.

For accuracy, the quantity of interest should have an uncertainty which is independent of the value of the quantity. As the uncertainty in an estimate of variance is approximately proportional to the variance, taking the logarithm of the variance yields a quantity with constant uncertainty (as is done, for example, in Ref. 31]). The studentized bootstrap yields additional accuracy, and involves normalizing by the estimated uncertainty in the quantity of interest 30. We have used both used the log scale and the studentized bootstrap in order to obtain accurate error bars.
[1] Caves, C. M. Quantum-mechanical noise in an interferometer. Phys. Rev. D 23, 1693-1708 (1981).

[2] Yurke, B., McCall, S. L. \& Klauder, J. R. SU(2) and $\mathrm{SU}(1,1)$ interferometers. Phys. Rev. A 33, 4033-4054 (1986).

[3] Giovannetti, V., Lloyd, S. \& Maccone, L. QuantumEnhanced Measurements: Beating the Standard Quantum Limit. Science 306, 1330-1336 (2004).

[4] Bollinger, J. J., Itano, W. M., Wineland, D. J. \& Heinzen, D. J. Optimal frequency measurements with maximally correlated states. Phys. Rev. A 54, R4649-R4652 (1996).

[5] Lee, H., Kok, P. \& Dowling, J. P. A quantum Rosetta stone for interferometry. J. Mod. Opt. 49, 2325-2338 (2002).

[6] Rarity, J. G. et al. Two-Photon Interference in a MachZehnder Interferometer. Phys. Rev. Lett. 65, 1348-1351 (1990).

[7] Fonseca, E. J. S., Monken, C. H. \& Pádua, S. Measurement of the de Broglie Wavelength of a Multiphoton Wave Packet. Phys. Rev. Lett. 82, 2868-2871 (1999).

[8] Edamatsu, K., Shimizu, R. \& Itoh, T. Measurement of the Photonic de Broglie Wavelength of Entangled Photon Pairs Generated by Spontaneous Parametric DownConversion. Phys. Rev. Lett. 89, 213601 (2002).

[9] Walther, P. et al. De Broglie wavelength of a non-local four-photon state. Nature 429, 158-161 (2004).

[10] Mitchell, M. W., Lundeen, J. S. \& Steinberg, A. M. Super-resolving phase measurements with a multiphoton entangled state. Nature 429, 161-164 (2004).

[11] Eisenberg, H. S., Hodelin, J. F., Khoury, G. \& Bouwmeester, D. Multiphoton Path Entanglement by Nonlocal Bunching. Phys. Rev. Lett. 94, 090502 (2005).

[12] Leibfried, D. et al. Creation of a six-atom 'Schrödinger cat' state. Nature 438, 639-642 (2005).

[13] Sun, F. W., Liu, B. H., Huang, Y. F., Ou, Z. Y. \& Guo, G. C. Observation of the four-photon de Broglie wavelength by state-projection measurement. Phys. Rev. A 74, 033812 (2006).

[14] Nagata, T., Okamoto, R., O’Brien, J. L., Sasaki, K. \& Takeuchi, S. Beating the Standard Quantum Limit with Four-Entangled Photons. Science 316, 726-729 (2007).

[15] Resch, K. J. et al. Time-Reversal and Super-Resolving Phase Measurements. Phys. Rev. Lett. 98, 223601 (2007).

[16] Kitaev, A. Y. Quantum measurements and the Abelian Stabilizer Problem. Electronic Colloquium on Computational Complexity 3, 3 (1996).

[17] Wiseman, H. M. Adaptive phase measurements of optical modes: Going beyond the marginal $Q$ distribution. Phys. Rev. Lett. 75, 4587-4590 (1995).

[18] Berry, D. W. \& Wiseman, H. M. Optimal States and 
Almost Optimal Adaptive Measurements for Quantum Interferometry. Phys. Rev. Lett. 85, 5098-5101 (2000).

[19] Armen, M. A., Au, J. K., Stockton, J. K., Doherty, A. C. \& Mabuchi, H. Adaptive homodyne measurement of optical phase. Phys. Rev. Lett. 89, 133602 (2002).

[20] Cook, R. L., Martin, P. J. \& Geremia, J. M. Optical coherent state discrimination using a closed-loop quantum measurement. Nature 446, 774-777 (2007).

[21] Mitchell, M. W. Metrology with entangled states. Proc. SPIE 5893, 589310 (2005).

[22] Nielsen, M. A. \& Chuang, I. L. Quantum Computation and Quantum Information (Cambridge Univ. Press, Cambridge, 2000).

[23] Rudolph, T. \& Grover, L. Quantum Communication Complexity of Establishing a Shared Reference Frame. Phys. Rev. Lett. 91, 217905 (2003).

[24] de Burgh, M. \& Bartlett, S. D. Quantum methods for clock synchronization: Beating the standard quantum limit without entanglement. Phys. Rev. A 72, 042301 (2005).

[25] Giovannetti, V., Lloyd, S. \& Maccone, L. Quantum Metrology. Phys. Rev. Lett. 96, 010401 (2006).

[26] van Dam, W., D’Ariano, G. M., Ekert, A., Macchiavello, C. \& Mosca, M. Optimal quantum circuits for general phase estimation. Phys. Rev. Lett. 98, 090501 (2007).

[27] Griffiths, R. B. \& Niu, C.-S. Semiclassical Fourier Transform for Quantum Computation. Phys. Rev. Lett. 76, 3228-3231 (1996).

[28] Wiseman, H. M. \& Killip, R. B. Adaptive single-shot phase measurements: A semiclassical approach. Phys. Rev. A 56, 944-957 (1997).

[29] Hradil, Z. et al. Quantum Phase in Interferometry. Phys. Rev. Lett. 76, 4295-4298 (1996).

[30] Davison, A. C. \& Hinkley, D. V. Bootstrap Methods and Their Application (Cambridge Univ. Press, Cambridge, 1997).

[31] Schenker, N. Qualms About Bootstrap Confidence Intervals. J. Am. Stat. Assoc. 80, 360-361 (1985).

Acknowledgements We thank M. Mitchell, D. Bulger and S. Lo for discussions. This work was supported by the Australian Research Council and the Queensland State Government.

Author information Correspondence should be addressed to G.J.P. (email: g.pryde@griffith.edu.au). 